\title{
Medical Management of Trigeminal Neuralgia
}

\author{
Subashree. $\mathrm{R}^{1}$ \\ Under Graduate ${ }^{I}$, Department of Oral Medicine and Radiology, Saveetha Dental College, India.
}

\begin{abstract}
This review discusses about the medical management of trigeminal neuralgia. Trigeminal neuralgia is the most common form of neuralgia. Management of trigeminal neuralgia is based on either on chemotherapy or on surgical management. Anticonvulsants are considered as most effective in the treating trigeminal neuralgia. Carbamazepine is considered as most efficient and primary drug for treating the disease. If mono therapy fails co-ordinations of drugs are followed. This review is relied on most representative and complete articles for this review article.
\end{abstract}

Keywords: Commonly used drugs, ill effect, Non surgical management, Trigeminal neuralgia.

\section{Introduction}

Trigeminal neuralgia is considered as one of the most severe painful disorder in the orofacial area[1]. It affects the trigeminal nerve. The main evidence for the pain is the compression of the trigeminal nerve root which is close to the entry of pons by an aberrant arterial or venous loop, other compressive lesions also cause pain this may be the secondary cause for the pain. The pain is unilateral in nature[2-3]. It persists from few seconds to minutes it might extend to hours, this occurs in bouts lasting in weeks with remission of periods in years. Each paroxysm of severe pain is followed by pain interval which stands for few minutes[4]. The pain is felt in different ways like electric shock, sharp-pricking, lancinating, excruciating, burning, stabbing and red hot needle like, forked lightening type of pain in the face[5]. Compression blood vessels may press on the trigeminal nerve as it leaves the brainstem at its cerebellopontine nerve root, compression of nerve root leads to demyelination. This results in spontaneous generation of electric impulses. This probably accounts for $90 \%$ of patients. In some patients it was thought to be a part of the ageing process as with increasing age the brain atrophies leading to redundant arterial loops which can cause compression. Other causes may be classified as idiopathic. It is also called as TIC-DOULOUREX[6-7]. This emphasizes the suddenness of pain which may be triggered with factors like cold or touch etc. This has got particular areas on the face called TRIGGER ZONES which precipitates pain on particular stimuli like washing face, shaving, winter season, brushing teeth etc. This characteristic pain affects four to five people in count of 10,000 . The aim of this review is to discuss about various drugs that are commonly used for the pain management of trigeminal neuralgia.

\section{Diagnostic Criteria}

This is based on the International Classification of Head ache and Disorders.

- Paroxysmal attacks of pain, lasting a second to two minutes and affecting one or more divisions of trigeminal nerve (typically maxillary or mandibular branches).

- Pain has atleast one of the following characteristics intense, sharp, superficial, stabbing, precipitated by trigger areas / factors .

- Attacks are similar in individual patients.

- There is no neurological deficit on examination.

- It is not caused by another disorder.

\section{Improving The Efficiency Of The Treatment}

In order to get the maximum treatment efficacy from drugs, patient's compliance is needed. Improved patient compliance can be achieved when the patient is explained about his illness completely by the doctor. When he/she is made to feel that they are the important part of the treatment. The benefits and adverse effect of each drug should be explained to the patient. The doctor should have extracted the complete history from the patient. Maintaining pain diaries helps to monitor the response to drugs.

\section{Commonly Used Drugs}

Anticonvulsants are most commonly used for the pain management. Carbamazepine is considered as first line of drug, inspite of its side effects. When this mono therapy fail, the second line of treatment is, combination of drugs like carbamazepine with lamotrigine or with baclofen. Other drugs are phenytoin, lamotrigine, gabapentin, oxcarbazepine, newer drugs like pregabalin[8]. 


\section{Anticonvulsants - Mode Of Action}

They exert multiple pharmacological actions which includes sodium channel blockade, calcium channel blockade, suppression of glutamate, action on NMDA/AMPA, action on GABA receptors.

\section{Role Of Drugs}

6.1.CARBAMAZEPINE: Carbamazepine is considered as first line of drug for the pain management of trigeminal neuralgia. It has onset of action within 24 hours. Side effects are greater compared to other drugs. It acts by stabilizing the inactivated state of voltage - gate sodium channels, making fewer of these channels available to subsequently open and this leaves the affected cells less excitable until the drug dissociates This drug has shown to potentiate GABA receptor made up alpha 1, alpha 2, gamma 2, subunits, thus it reduces in neuropathic pain[9] DOSAGE:400mg/day

6.2.OXCARBAZEPINE: This drug considered as the substitute for carbamazepine, this drug is used when there is increased adverse effects \& poor efficacy produced by carbamazepine. It is 10,11 , dihydro-10-oxo- $5 \mathrm{H}-$ dibenzapine 5-carboxaminade, keto derivative of carbamazepine. Shows reduced side effects compared to carbamazepine rapidly absorbed after oral ingestion. Oxcarbazepine and its, active metabolite, mono hydroxy derivative, have effects and blocks the sodium gated channels \& possibly potassium \& calcium channels, and results in stabilization of hyper excite \& neural members, inhibition of repetitive neuronal firing \& propagation of synaptic impulses thus produces, anticonvulsants effects[10]. DOSAGE: 600-2400mg/day.

6.3.PHENYTOIN: It is an anti-epileptic drug, also used as anticonvulsants. It is 5-5diphenylhydantoin. It's one of the drugs used in treatment of trigeminal neuralgia. It is second line of drugs. This drug has the capacity to block sustained high frequency repetitive firing of action potentials. This is accomplished by reducing the amplitude of sodium dependent action through enhancement of steady state inactivation .It binds preferentially to inactive form of the channel. Because it takes time for bound drug to dissociate form the in active channel. They are time dependent to block the channel. Since the fraction of inactive channels is increased by membrane depolarization as well as by repetitive firing[11]. This binding with the inactive state phenytoin sodium can produce voltage-use-\&time dependent block of sodium dependent action potential.DOSAGE:300-600mg/day.

6.4.LAMOTRIGINE: Lamotrigine is an anti-epileptic drug. It is also used as anticonvulsant. This drug is generally accepted as sodium channel blockers with additional actions since these drugs have broad spectrum action. This acts by stabilizing the neuronal membrane and consequently modulating pre-synaptic transmitter and releases the excitatory amino acids.DOSAGE:400-1200mg/day.

6.5.BACLOFEN: It is a muscle relaxant, also used as anticonvulsants. This drug acts by activating the GABAb receptors as it does not have significant affinity for GHB receptors the modulation of GABAb receptors is what produces its range of therapeutic properties[1] DOSAGE:40-80mg/day.

6.6.GABAPENTIN: This drug is commonly used to treat nerve pain .It was started to treat trigeminal neuralgia in the year 1994.This drug acts with the cortical neurons at auxiliary subunits of voltage sensitive calcium channels. It increases the synaptic cone of GABA, enhance the GABA response at non synaptic sites in neuronal tissues. Thus reduces the release of mono amino neurotransmitters. This drug shows anti hyperanalgesic \& antiallodymic effects by reducing noradrenergic system[12]DOSAGE:100-300 mg/day.

6.7.PREGABLIN: This is structurally similar to gabapentin with recognized efficacy in the treatment of neuropathic pain. The pharmacological mechanism by which these agents exert their clinical effects have, until recently,remained unclear. The interaction of gabapentin and pregabalin with conventional antiepileptic and analgesic drug is likely to be modest and been largely dismissed in favor of a selective inhibitory effect on voltage gated calcium channels containing the alpha 1 subunit. This mechanism is consistently observed in the both rodent -human based experimental paradigms and be sufficiently robust to account for much of the clinical activity of these compounds[13]

\section{Discussion:}

Anticonvulsants are usually considered one of the most efficient and effective drug for the treating trigeminal neuralgia pain. Studies shows that carbamazepine is one the most common and primary drug used for treating trigeminal neuralgia. 
Tomson et al[14] studied in detail about the diurnal pain distribution and its relation to drug carbamazepine dosing and plasma concentration and the effect of drug and the dose. Trigeminal neuralgia pain distribution showed marked intra individual with pain free nights and increased in the drop of pain during the mid day hours this later coincided in time with the peak plasma concentration of carbamazepine thus indicating the effect of plasma concentration and the fluctuations on pain management. Mac quay et al[15] stated that carbamazepine had the combined drug for effective treatment.

Fargo[16] tested the Dihydroketo and dihydromono hydroxyl derivatives analogues of carbamazepine for efficiency and tolerability in trigeminal neuralgia, both the derivatives brought about freedom from the symptoms or a marked reduction in the pain in all the patients. The onset of action was observed in next 48 hours, in most of the cases. For both analogues the effective dose was between 10 and $20 \mathrm{mg} / \mathrm{kg}$ body weight in most of the patients. There was a linear relationship, with a correlation co efficient of 0.83 between the doses and the serum level. Doses almost twice as high as the dose. Carbamazepine is needed to achieve freedom the signs and symptoms with carbamazepine analogues. Because of the adverse effects in the form of dizziness and ataxia occur much less frequently than with carbamazepine. The analogues can be administrated in the higher doses.

Koyama et al[17] in his study on 66 patients suggested that the biochemical parameters such as levels of albumin as showed a significant relationship with carbamazepine free relation. Non glycated albumin was more correlated strongly with carbamazepine free reaction. This fraction was strongly correlated with age of the individual. Where the albumin ligands of carbamazepine in the serum was non glycated albumin were decreased with age, albumin and glycated albumin and carbamazepine free fraction which is much higher in the elderly than in patients less than 65 years old of age. These suggested that in the elderly patients the elevators of the free carbamazepine concentration in the serum caused by reduced non glycated albumin levels induces increases in the sensitivity of the pharmacologic actions of carbamazepine and the risk of drug interactions.

Merren[18] stated that gabapentin is an alternative drug for the treatment of trigeminal neuralgia and it does not affect the metabolism and other medication pathway. This drug has well tolerability and fast onset of action.

Zakrewaska et al[19] suggested that lamotrigine is another choice of drug for treating trigeminal neuralgia, it has anti neuralgic properties. He stated that this drug can used for treating refractory trigeminal neuralgia, and mainly it is used as maintain dose it's about $400 \mathrm{mg}$. lamotrigine was considered superior to placebo, adverse effects was only dose dependent.

Clonazepam seems to be another effective drug for treating idiopathic trigeminal neuralgia but this drug shows increased drowsiness. Oxcarbazepine is a potent anti neuralgic drug and possess good acceptability and tolerability, it is also used along carbamazepine dosage and this is gradually increased up to higher efficiency and increased therapeutic effects. Comparatively decreased adverse effects seen.

Gibron et al[20] studied response of topiramate in patients, all of the patients responded to this drug better in percentage of $31,42,63 \%$.

\section{Adverse Effects:}

Patients exhibits wide range of side effects in the initial stage of treatment with anticonvulsants for trigeminal neuralgia. Carbamazepine possesses side effects like dizziness, ataxia, drowsiness and reduction of alertness occurs and these effects seem to be increased with stable doses on doses[1].

Delcker et al[21] quantified the effect of carbamazepine on postural stability by petrography. It shows the composition equilibrium score scored significant reduction of postural stability with increase in the relation to dose of carbamazepine. This was concluded that rate of change of carbamazepine doses in an important determinant of cognitive and motor functions in the increasing dose phase.

Kramlinger et al[22] stated carbamazepine is a safe drug comparatively and well tolerated with rare side effects like hematologic activity which began from rashes to life threatening fumigant eruptions.

\section{Conclusion:}

Anticonvulsants are effective in the treatment of trigeminal neuralgia. Carbamazepine and oxcarbazepine are considered as the first choice of drugs, lamotrigine is a potent drug in treating refractory trigeminal neuralgia. Adequate studies have not been done for oxcarbazepine when compared with carbamazepine, but the efficacy of these drugs appears similar and oxcarbazepine is better tolerated than carbamazepine. Hence, more clinical trials have to be performed to determine the efficacy of oxcarbazepine over carbamazepine in the future. 


\section{References}

[1]. Jens c.Torp, Dr. Med Dent and John P.Gobbetlin, school of dentistry Michigan Trigeminal neuralgia versus atypical facial pain, a review of literature and case report. Oral surgery and Med Pathol Radio Endod 1996;81;424-32.

[2]. Fromm GO. Sessele BJ summary and conclusion. In Fromm GH, Sessele Trigeminal neuralgia concepts regarding pathogenesis and treatment. Boston butterworth Heinemann 1991;205-19

[3]. Sprotte G.Gesichtsschmez In ZenzM, Jurna I eds,Lehrbuch der Schmerztherapie. Stuttgart. Weissenchafliche, Verlagsgesellschaft 1993;405-16.

[4]. Murskey H.Bodguk, Classification on chronic pain $2^{\text {nd }}$ ed. Seattle LASP Press 1994;57-60.

[5]. Loser JD Tic-douloureux and atypical facial pain. Wall PD Melzack, eds, Textbook of pain $3^{\text {rd }}$ ed, Edinburgh Chruchill Livingston.1994;699-710.

[6]. Buurchiel K.J. Trigeminal neuropathic pain. Acta neurochir supplement.1993;58;148-9.

[7]. Jannetta PJ. Arterial compression of trigeminal nerve at the pons in patients with trigeminal neuralgia . J. Neurosurgery $1967 ; 26 ; 159-62$.

[8] Marco Pappagallo; Newer antiepileptic drugs; Possible uses in the treatment of neuropathic pain and maigraine. clinical therapeutics; vol:25 No:10,2003. review. BMJ 1995;311:1047-52.

[12]. Parke davis, Division of Warnet lambat company, Morris ,Plaines, New Jersy. Non epileptic uses of gabapentin.vol:40:566572.1999 .

[13]. M.Obermann,MSYoon,K.Seven,MMascheke,HC.DienerandZ.katsarava, Efficiency of pregabalin in the treatment of trigeminal neuralgia. Doi:10 1111/j1468-298[9].J.C.Taylor, Susan Bracer,M.L.E.Espir.long term treatment of trigeminal neuralgia with carbamazepine.1981:57;16-18.

[10]. JM.Zakrzewska,PN.Patslos.Journal of neurology,Psychaitary 1989;52:472-476.

[11]. HenryMc.Quay, DawnCaroll, AlefandroRJadad, Phillipwiffin, AndrewMoore. Anticonvulsants drugs for management fo pain a systemic 2.2007 .

[14]. Tomson T, Ekbom K: Trigeminal neuralgia: Time course of pain in relation to carbamazepine dosing Cephalalgia 1:91, 1981

[15]. McQuay H, Carroll D, Jadad AR, et al: Anticonvulsant drugs for management of pain: A systematic review . BMJ 311:1047, 1995 .

[16]. Farago F: Trigeminal neuralgia: Its treatment with two new carbamazepine analogues. Eur Neurol 26:73, 1987

[17]. Koyama H, Sugioka N, Uno A, et al: Age-related alteration of carbamazepine-serum protein binding in man . $J$ Pharm Pharmacol 51:1009, 1999

[18]. Merren MD: Gabapentin for treatment of pain and tremor: A large case series. South Med J 91:739, 1998

[19]. Zakrzewska JM, Chaudhry Z, Nurmikko TJ, et al: Lamotrigine (Lamictal) in refractory trigeminal neuralgia: Results from adoubleblind placebo controlled crossover trial. Pain 73:223,1997.

[20]. Gilron I, Booher SL, Rowan JS, et al: Topiramate in trigeminal neuralgia: a randomized, placebo-controlled multiple crossover pilot study. Clin Neuropharmacol 24:109, 2001.

[21]. Delcker A, Wilhelm H, Timmann D, et al: Side effects from increased doses of carbamazepine on neuropsychological and posturographic parameters of humans. Eur Neuropsychopharmacol 7:213, 1997.

[22]. Kramlinger KG, Phillips KA, Post RM: Rash complicating carbamazepine treatment. J Clin Psychopharmacol 14:408, 1994. 